\title{
Space-Dependent Dirac Operators and Effective Quantum Field Theory for Fermions ${ }^{\star}$
}

\author{
John Z. Imbrie ${ }^{1 \star \star}$, Steven A. Janowsky ${ }^{1 \star \star \star}$ and Jonathan Weitsman ${ }^{1,2 \star \star \star \star}$ \\ ${ }^{1}$ Harvard University, Departments of Mathematics and Physics, Cambridge, MA 02138, USA \\ 2 M.I.T., Department of Mathematics, Cambridge, MA 02139, USA
}

Received June 9, 1989; in revised form May 17, 1990

\begin{abstract}
For the operator $i \not \partial+m(x)$, where $m(x)$ can change sign, we develop a cluster expansion for computing the determinant and Green's functions. We use a local chiral transformation to relate the space-dependent case to the ordinary Dirac operator.
\end{abstract}

\section{Introduction}

The study of multi-phase field theories with generalized Yukawa interactions provides a natural structure for studying Dirac operators with space-dependent mass. Different phases of such a model will have different effective fermion masses. If one attempts to analyze such a model via a cluster expansion, different cluster will be in different phases and have different masses for the fermions.

Our specific motivation for studying operators like $i \not \partial+m(x)$ comes from trying to understand the phase structure of two-dimensional Wess-Zumino models. While the single phase case has been studied [15] and much is known for the system in finite volume [9-12], the infinite volume multiphase problem remains unexplored.

A first step to understanding the behavior of the Wess-Zumino model is to study a simpler toy model with almost no bosonic field. By "almost no" field we mean that the only remnant of the boson is a restriction that each block of spacetime is in a particular phase. This results in the study of a Dirac operator $i \not \partial+m(x)$ in two dimensions where $m(x)$ takes on a small number of values.

Dirac Operators with Space Dependent Masses. To obtain a view of the technical

* Supported in part by National Science Foundation grant PHY/DMS 88-16214

$\star \star$ Alfred P. Sloan Foundation Fellow

$\star \star \star$ Supported in part by National Science Foundation grants DMS 90-08827 and DMS $88-58073$

$\star \star \star \star$ Supported in part by National Science Foundation Mathematical Sciences Postdoctoral Research Fellowship DMS 88-07291 
problems involved, consider first the Laplacian with a space dependent potential

$$
-\Delta+m^{2}(x)
$$

where

$$
m_{0} \geqq m(x)>\varepsilon>0 .
$$

Exponential decay for the Green's function $\left(-\Delta+m^{2}(x)\right)^{-1}$ can be established using the Neumann series

$$
\begin{aligned}
\left(-\Delta+m^{2}(x)\right)^{-1} & =\left[1+\left(-\Delta+m_{0}^{2}\right)^{-1}\left(m^{2}(x)-m_{0}^{2}\right)\right]^{-1}\left(-\Delta+m_{0}^{2}\right)^{-1} \\
& =\sum_{k=0}^{\infty}\left[\left(-\Delta+m_{0}^{2}\right)^{-1}\left(m_{0}^{2}-m^{2}(x)\right)\right]^{k}\left(-\Delta+m_{0}^{2}\right)^{-1}
\end{aligned}
$$

where convergence is guaranteed since $\left|m_{0}^{2}-m^{2}(x)\right|<m_{0}^{2}$. by

Now consider the Dirac operator $i \not \phi+m(x)$ on $\mathbb{R}^{2}$. This is the operator defined

$$
i \not \partial=i \gamma_{0}^{E} \partial_{x_{0}}+i \gamma_{1}^{E} \partial_{x_{1}}
$$

where $\gamma_{0}^{E}, \gamma_{1}^{E}$ are $2 \times 2$ matrices

$$
\gamma_{0}^{E}=\left(\begin{array}{rr}
0 & -1 \\
1 & 0
\end{array}\right), \quad \gamma_{1}^{E}=\left(\begin{array}{ll}
0 & i \\
i & 0
\end{array}\right) .
$$

The Neumann series technique described above again works so long as $m(x)$ satisfies a bound of the form (1.1). However in this case of the Dirac operator the restriction to positive mass is no longer trivial; in fact the effective quantum field theories of $[11,12]$ give rise to Dirac operators with $m(x)$ taking different signs in different space time regions.

In order to handle this problem we begin by remarking that the operators $i \not \varnothing+m$ and $i \not \partial-m$ are related via a (chiral) unitary transformation by $\gamma_{5}=\gamma_{0} \gamma_{1}$, where $\gamma_{0}=i \gamma_{0}^{E}$ and $\gamma_{1}=\gamma_{1}^{E}$ :

$$
\gamma_{5} \gamma_{0}(i \not \partial+m) \gamma_{5}=\gamma_{0}(i \not \partial-m) .
$$

Similarly we have, with $U=e^{i \alpha(x) \gamma_{5}}, \alpha \in C_{0}^{\infty}\left(\mathbb{R}^{2}\right)$,

$$
U^{-1} \gamma_{0}(i \not \partial+m) U=\gamma_{0}\left(i \not \partial+e^{2 i \alpha(x) \gamma_{5}} m-\not \partial \alpha(x) \gamma_{5}\right) .
$$

If we choose $\alpha(x)=0$ in one region (region $A$ ), and $\alpha(x)=\pi / 2$ in region $B$ (with a boundary layer between), then

$$
U^{-1} \gamma_{0}(i \not \partial+m) U= \begin{cases}\gamma_{0}(i \not \partial+m), & \text { in region } A \\ \gamma_{0}(i \not \partial-m), & \text { in region } B .\end{cases}
$$

Thus the Dirac operator with masses of differing signs is related to the Dirac operator with constant mass via a unitary transformation.

This is our main technical device for handling the Dirac operator with differing signs for the masses. For simplicity we will consider the case where the function $m(x)= \pm 1$. We discuss the more general case in the appendix, where we combine our methods with a Neumann series of the type discussed above (1.2). We could also easily consider the case where the mass was chiral, i.e. proportional to 
$\cos \theta+i \gamma_{5} \sin \theta, \theta \in \mathbb{R}$. Although we consider only the two dimensional case, we believe an extension of our methods should work in any even dimension.

Before stating our main theorem, however, we must make the following remark. Let $\gamma_{0}(i \not \partial+m(x))$ be the Dirac operator on $\mathscr{H}_{1 / 2} \oplus \mathscr{H}_{1 / 2}$, where $\mathscr{H}_{\alpha}$ is the Sobolev space $\mathscr{H}_{\alpha}=L^{2}\left(\mathbb{R}^{2},\left(p^{2}+1\right)^{\alpha} d^{2} p\right)$. Let $S=\left(\gamma_{0}(i \not \partial+1)\right)^{-1}$ be the operator on $\mathscr{H} \equiv \mathscr{H}_{-1 / 2} \oplus \mathscr{H}_{-1 / 2}$ determined by the kernel

$$
S(x, y)=\int \frac{-\not p+1}{p^{2}+1} e^{-i p(x-y)} \frac{d^{2} p}{(2 \pi)^{2}} \gamma_{0},
$$

i.e. the kernel (2.5) without cutoff. We wish to prove estimates on the putative Green's function

$$
\left[\gamma_{0}(i \not \partial+m(x))\right]^{-1} \text {. }
$$

In fact it is not difficult to write down functions $m(x)$ for which this inverse will not exist. To circumvent this problem we compute the "reduced" Green's function, formally given by

$$
\left[\gamma_{0}(i \not \partial+m(x))\right]^{-1} \operatorname{det}_{3}\left(S\left[\gamma_{0}(i \not \partial+m(x))\right]\right)=(1+K)^{-1} S \operatorname{det}_{3}(1+K),
$$

where $K=S \gamma_{0}(m(x)-1)$ is an operator on $\mathscr{H}$. The object $(1.10)$ is well defined and given by a Fredholm series [14], whether or not $\gamma_{0}(i \not \partial+m(x))$ is invertible. Convergence of the Fredholm series is guaranteed by Lemma 2.1. Note that where $\gamma_{0}(i \not \partial+m(x))$ is invertible, the reduced Green's function differs from the inverse by an irrelevant constant.

We may now state our main theorem.

Theorem 1. Let $g, h \in C_{0}^{\infty}\left(\mathbb{R}^{2}\right)$ be supported in unit squares $\Delta_{g}, \Delta_{h}$. Then there exists $c>0$ such that

$$
\begin{aligned}
& \left|\left\langle g,(1+K)^{-1} S \operatorname{det}_{3}(1+K) h\right\rangle\right| \\
& \quad \leqq\|g\|_{\mathscr{H}}\|h\|_{\mathscr{H}} \exp \left[-c d\left(\Delta_{g}, \Delta_{h}\right)\right] \exp \left[2\left|\Lambda_{-1}\right| / \pi+O\left(\left|\partial \Lambda_{-1}\right|\right)\right],
\end{aligned}
$$

where

$$
\Lambda_{-1}=\operatorname{supp}(1-m(x)) \text {. }
$$

A similar result holds for higher Fredholm minors. We also have the following estimate for the regularized determinant (Proposition 2.7):

$$
\left|\operatorname{det}_{3}(1+K)\right| \leqq \exp \left[2\left|\Lambda_{-1}\right| / \pi+O\left(\left|\partial \Lambda_{-1}\right|\right)\right]
$$

where the coefficient of $\left|\Lambda_{-1}\right|$ has the expected perturbative value.

The main idea of the proof is to make use of the unitary transformation $U$ to relate $\gamma_{0}(i \not \partial+m(x))$ to an operator equal to $\gamma_{0}(i \not \partial+1)+\zeta$, where $\zeta$ is supported on $\partial \Lambda_{-1}$. The correction $2\left|\Lambda_{-1}\right| / \pi$ arises since, unlike a true determinant, $\operatorname{det}_{3}$ is not invariant under unitary conjugation.

This result gives the main contribution to the weights of the contour expansion for the multiphase $N=2$ Wess-Zumino 2 models. We would hope that a slightly different version of this work, replacing determinants by Pfaffians, should give a similar result for the $N=1$ models. The cluster expansion for these quantum field theories will be the subject of future papers [8]. For the reader interested in the quantum field theories this paper should serve as a simple introduction to the 
necessary cluster expansion techniques. We hope however that this result may be of interest in itself.

\section{Estimates for the Determinant}

Before turning to estimates on Fredholm minors, let us consider the determinant alone. We want to be able to compute determinant ratios, such as that formally given by

$$
\operatorname{det}_{3} \gamma_{0}(i \not \partial+m(x)) / \operatorname{det}_{3} \gamma_{0}(i \not \partial+1)
$$

The existence of the determinant is guaranteed by the following, which is an immediate consequence of Lemma 5.4:

Lemma 2.1. Let $\mathscr{L}_{3}(\mathscr{H})$ denote the 3 -Schatten ideal, i.e. the ideal of operators $\mathscr{K}$ on $\mathscr{H}$ such that

$$
\operatorname{Tr}\left(\mathscr{K}^{*} \mathscr{K}\right)^{3 / 2}<\infty
$$

Then

$$
K \in \mathscr{L}_{3}(\mathscr{H}) .
$$

The fact that it is regularized determinants which appear complicates our analysis. While (formally)

$$
\frac{\operatorname{det} \gamma_{0}(i \not \partial-1)}{\operatorname{det} \gamma_{0}(i \not \partial+1)}=\operatorname{det}\left(1-2 S \gamma_{0}\right)=1
$$

because of (1.5), we (unfortunately) have

$$
\operatorname{det}_{3}\left(1-2 S \gamma_{0}\right)=\exp \lim _{\kappa \rightarrow \infty} T_{\kappa}
$$

where

$$
T_{\kappa}=\operatorname{Tr}\left[2 S_{\kappa} \gamma_{0}+2 S_{\kappa} \gamma_{0} S \gamma_{0}\right]
$$

here $S_{\kappa}$ is the operator on $\mathscr{H}_{-1 / 2} \oplus \mathscr{H}_{-1 / 2}$ given by the kernel

$$
S_{\kappa}(x, y)=\int \frac{-p+1}{p^{2}+1} e^{-i p(x-y)} \chi_{\kappa}(p) \frac{d^{2} p}{(2 \pi)^{2}} \gamma_{0},
$$

where

$$
\chi_{\kappa}(p)=e^{-p^{2} / \kappa^{2}}
$$

Let $\Xi_{\kappa}$ be the operator on $\mathscr{H}$ defined by

$$
\Xi_{\kappa} f=\int \chi_{\kappa}(p) \widehat{f}(p) \frac{d^{2} p}{(2 \pi)^{2}} .
$$

Then $S_{\kappa}=\Xi_{\kappa} S=S \Xi_{\kappa}$.

It is easily seen that in a finite volume $\Lambda$

$$
\lim _{\kappa \rightarrow \infty} T_{\kappa}=2|\Lambda| / \pi+O(|\partial \Lambda|) .
$$


Thus in the local case we expect (finite) $O(|\Lambda|)$ corrections from the difference between det and $\operatorname{det}_{3}$, as well as boundary corrections.

Let the function $m(x)$ take the values \pm 1 ; let

$$
\zeta=\gamma_{0}\left(m(x) e^{2 i \alpha \gamma_{5}}-1-\not \alpha \gamma_{5}\right)
$$

where we choose $\alpha$ such that $m(x) e^{2 i \alpha(x)}=1$ except in a neighborhood of $\partial \Lambda_{-1}$. Let

$$
R_{\kappa}=\operatorname{Tr} \Xi_{\kappa}\left[S \gamma_{0}(1-m(x))+\left(S \gamma_{0}(1-m(x))^{2} / 2\right]+\operatorname{Tr} \Xi_{\kappa}\left[S \zeta-(S \zeta)^{2} / 2\right]\right.
$$

Then we have the following:

\section{Proposition 2.2}

(a) Then limit $\lim _{\kappa \rightarrow \infty} R_{\kappa}$ exists, and

$$
R \equiv \lim _{\kappa \rightarrow \infty} R_{\kappa}=2\left|\Lambda_{-1}\right| / \pi+O\left(\left|\partial \Lambda_{-1}\right|\right)
$$

(b) The determinant $\operatorname{det}_{3}\left[1+S \gamma_{0}(m(x)-1)\right]$ satisfies

$$
\operatorname{det}_{3}\left[1+S \gamma_{0}(m(x)-1)\right]=\operatorname{det}_{3}[1+S \zeta] \exp (R+r),
$$

where

$$
r=\frac{1}{4 \pi}\|\partial \alpha\|_{L^{2}}^{2} .
$$

Remark. The factor $\exp R$ is the ratio of the determinants expected from a naive product formula. The additional term exp $r$ is a correction resulting from the need to place cutoffs on operators before such a formula can apply.

Proof. Part (a) follows by a straightforward calculation. We devote the remainder of this section to proving (b). We begin with the following lemma:

Lemma 2.3. Let $A, B \in \mathscr{L}_{3}(\mathscr{H})$. Then

$$
C=A+B+A B=(1+A)(1+B)-1 \in \mathscr{L}_{3}(\mathscr{H})
$$

and

$$
\operatorname{det}_{3}(1+A) \operatorname{det}_{3}(1+B)=\operatorname{det}_{3}(1+C) \exp -\operatorname{Tr} T,
$$

where

$$
T=(A B)^{2} / 2+A B^{2}+B A^{2} \in \mathscr{L}_{1}(\mathscr{H}) .
$$

Proof. If $A, B \in \mathscr{L}_{1}(\mathscr{H})$, then

$$
\operatorname{Tr} T=\operatorname{Tr}\left[A+B-C-A^{2} / 2-B^{2} / 2+C^{2} / 2\right],
$$

and the lemma follows by the product formula for Fredholm determinants and the definition of $\operatorname{det}_{3}$. Since operators in $\mathscr{L}_{3}(\mathscr{H})$ may be approximated by finite rank operators, the lemma follows.

We now apply the above lemma to our case. Let $U_{t}=\exp \left[i \alpha \gamma_{5} t\right]$, and let

$$
\zeta_{t}=\gamma_{0}\left(m(x) e^{2 i \alpha \gamma_{5} t}-1-\not \alpha \alpha \gamma_{5} t\right)
$$


Then

$$
\begin{aligned}
1+S \zeta_{t} & =S U_{t}^{-1} \gamma_{0}(i \not \partial+m(x)) U_{t} \\
& =S U_{t}^{-1} S^{-1} U_{t} U_{t}^{-1} S \gamma_{0}(i \not \partial+m(x)) U_{t} \\
& =\left(1+S \zeta_{t}^{0}\right)\left(1+U_{t}^{-1} K U_{t}\right)
\end{aligned}
$$

where

$$
\zeta_{t}^{0}=\gamma_{0}\left(e^{2 i \alpha \gamma_{5} t}-1-\not \alpha \gamma_{5} t\right) .
$$

As a consequence of Lemma 2.3 we have the following:

Corollary 2.4. Let

$$
T_{t}=\left(S \zeta_{t}^{0} U_{t}^{-1} K U_{t}\right)^{2} / 2+\left(S \zeta_{t}^{0}\right)^{2} U_{t}^{-1} K U_{t}+\left(U_{t}^{-1} K U_{t}\right)^{2} S \zeta_{t}^{0} .
$$

Then

$$
\begin{aligned}
\operatorname{det}_{3}\left[1+S \zeta_{t}\right] & =\operatorname{det}_{3}\left[1+S \zeta_{t}^{0}\right] \operatorname{det}_{3}\left[1+U_{t}^{-1} K U_{t}\right] \exp \operatorname{Tr} T_{t} \\
& =\operatorname{det}_{3}\left[1+S \zeta_{t}^{0}\right] \operatorname{det}_{3}[1+K] \exp \operatorname{Tr} T_{t} .
\end{aligned}
$$

On the other hand, some algebraic manipulation shows that

$$
\begin{aligned}
\operatorname{Tr} T & \left.\equiv \operatorname{Tr} T_{t}\right|_{t=1} \\
& =\lim _{\kappa \rightarrow \infty} \operatorname{Tr} \Xi_{\kappa} T \\
& =\lim _{\kappa \rightarrow \infty} \operatorname{Tr}\left(R_{\kappa}+E_{\kappa}+F_{\kappa}\right),
\end{aligned}
$$

where

$$
E_{\kappa}=\left.E_{\kappa, t}\right|_{t=1}, \quad F_{\kappa}=\left.F_{\kappa, t}\right|_{t=1}
$$

with

$$
E_{\kappa, t}=\operatorname{Tr} \Xi_{\kappa}\left(S \zeta_{t}^{0}-\left(S \zeta_{t}^{0}\right)^{2} / 2\right)
$$

and

$$
F_{\kappa, t}=\frac{1}{2} \operatorname{Tr} \Xi_{\kappa}\left[S \zeta_{t}^{0}, U_{t}^{-1} K U_{t}\right]
$$

The term $R_{\kappa}$ was estimated in Proposition 2.2, part (a). For $E_{\kappa, t}$ and $F_{\kappa, t}$ we have the following results:

\section{Lemma 2.5 .}

(a) $E(t)=\lim _{\kappa \rightarrow \infty} E_{\kappa, t}$ exists, and

$$
\operatorname{det}_{3}\left[1+S \zeta_{t}^{0}\right] \exp E(t)=\exp \frac{-t^{2}}{4 \pi}\|\partial \alpha\|_{2}^{2}
$$

(b) $\lim _{\kappa \rightarrow \infty} F_{\kappa, t}=0$.

To prove this we make use of the following lemma:

Lemma 2.6 The operator $U_{t}^{-1} \Xi_{\kappa} U_{t}$ may be written as a sum

$$
U_{t}^{-1} \Xi_{\kappa} U_{t}=\Xi_{\kappa}+\mathcal{O}_{\kappa}^{5} \gamma_{5}+\mathcal{O}_{\kappa}^{I},
$$


where for any $\varepsilon>0$,

$$
\begin{array}{lll}
\left\|\mathcal{O}_{\kappa}^{I}\right\|_{1+\varepsilon} \rightarrow 0 & \text { as } & \kappa \rightarrow \infty, \\
\left\|\mathcal{O}_{\kappa}^{5}\right\|_{2+\varepsilon} \rightarrow 0 & \text { as } & \kappa \rightarrow \infty .
\end{array}
$$

Proof. We first demonstrate (2.30). The operator $\mathcal{O}_{\kappa}^{5}$ is given by the kernel

$$
\mathcal{O}_{\kappa}^{5}(x, y)=\frac{i \gamma_{5} \kappa^{2}}{4 \pi} \sin (\alpha(x)-\alpha(y)) e^{-\kappa^{2}(x-y)^{2} / 4} .
$$

We write $\mathcal{O}_{\kappa}^{5}=L_{1}^{5}+L_{2}^{5}$, where $L_{1}^{5}, L_{2}^{5}$ are given by the kernels

$$
\begin{aligned}
& L_{1}^{5}(x, y)=\frac{i \gamma_{5} \kappa^{2}}{4 \pi} \partial \alpha(x) \cdot(x-y) e^{-\kappa^{2}(x-y)^{2} / 4}, \\
& L_{2}^{5}(x, y)=\mathcal{O}_{\kappa}^{5}(x, y)-L_{1}^{5}(x, y) .
\end{aligned}
$$

We have

$$
\left\|\mathcal{O}_{\kappa}^{5}\right\|_{2+\varepsilon} \leqq\left\|L_{1}^{5}\right\|_{2+\varepsilon}+\left\|L_{2}^{5}\right\|_{2} .
$$

Using the notation of Lemma 2.1 of [13], $L_{1}^{5}$ is of the form

where

$$
\text { const } \times f(p) g(x) \text {, }
$$

Thus

$$
\begin{aligned}
& f(p)=p \kappa^{-2} e^{-p^{2} / \kappa^{2}}, \\
& g(x)=\partial \alpha(x) .
\end{aligned}
$$

$$
\begin{aligned}
\left\|L_{1}^{5}\right\|_{2+\varepsilon} & \leqq \text { const } \times\|f\|_{L^{2+\varepsilon}\left(\mathbb{R}^{2}\right)}\|g\|_{L^{2+\varepsilon}\left(\mathbb{R}^{2}\right)} \\
& \leqq O\left(\left|\partial \Lambda_{-1}\right|\right) \kappa^{-\varepsilon /(2+\varepsilon)}
\end{aligned}
$$

The Hilbert-Schmidt norm of $L_{2}^{5}$ is easily estimated using pointwise bounds, yielding (2.30).

To deal with (2.29) we must get around the fact that Lemma 2.1 of [13] is not directly applicable to $(1+\varepsilon)$-norms. The kernel of $\mathcal{O}_{\kappa}^{I}$ is given by

$$
\mathcal{O}_{\kappa}^{I}(x, y)=(\cos (\alpha(x)-\alpha(y))-1) \frac{\kappa^{2}}{4 \pi} e^{-\kappa^{2}(x-y)^{2} / 4} .
$$

Let $\chi$ be the characteristic function of $\Lambda_{-1}$. Then

and

$$
\mathcal{O}_{\kappa}^{I}=\chi \mathcal{O}_{\kappa}^{I}+\mathcal{O}_{\kappa}^{I} \chi-\chi \mathcal{O}_{\kappa}^{I} \chi,
$$

$$
\left\|\mathcal{O}_{\kappa}^{I}\right\|_{1+\varepsilon} \leqq 3\left\|\chi \mathcal{O}_{\kappa}^{I}\right\|_{1+\varepsilon} .
$$

Now we write

Now

$$
\begin{aligned}
\left\|\chi \mathcal{O}_{\kappa}^{I}\right\|_{1+\varepsilon} & \leqq\left\|\chi S \gamma_{0}(i \not \partial+1) \mathcal{O}_{\kappa}^{I}\right\|_{1+\varepsilon} \\
& \leqq\|\chi S\|_{2+\delta}\left\|(i \not \partial+1) \mathcal{O}_{\kappa}^{I}\right\|_{2+\delta} \\
& \leqq \text { const } \times\left\|(i \not \partial+1) \mathcal{O}_{\kappa}^{I}\right\|_{2+\delta}
\end{aligned}
$$

$$
(i \not \partial+1) \mathcal{O}_{\kappa}^{I}(x, y)=K_{1}^{I}(x, y)+K_{2}^{I}(x, y),
$$


where the leading term is

$$
K_{1}^{I}(x, y)=\frac{-i \kappa^{2}}{4 \pi} \partial \alpha(x) \cdot(x-y) \partial \alpha(x) e^{-\kappa^{2}(x-y)^{2}},
$$

the kernel of an operator $K_{1}^{I}$ whose $(2+\delta)$-norm may be estimated similarly to (2.38). The remainder $K_{2}^{I}(x, y)$ is the kernel of an operator whose Hilbert-Schmidt norm vanishes as $\kappa \rightarrow \infty$, yielding the lemma.

Proof of Lemma 2.5. To show (b), we note that

$$
F_{\kappa, t}=\frac{1}{2} \operatorname{Tr} S\left(\Xi_{\kappa} \zeta_{t}^{0}-\zeta_{t}^{0} \Xi_{\kappa}\right)\left(U_{t}^{-1} K U_{t}\right)
$$

This can be expressed as a sum of terms, some of which are of the form $\operatorname{Tr}\left(\Xi_{\kappa} \zeta_{t}^{0}-\zeta_{t}^{0} \Xi_{\kappa}\right) \mathcal{O}$, with $\mathcal{O} \in \mathscr{L}_{1}$, and hence vanish as $\kappa \rightarrow \infty$. The remaining terms may be estimated by

$$
\int \frac{1}{\left(p^{2}+1\right)^{1 / 2}} \frac{1}{\left(q^{2}+1\right)^{1 / 2}}|\widehat{f}(p-q)|(1-m(y))\left|\chi_{\kappa}(p)-\chi_{\kappa}(q)\right| d^{2} p d^{2} q d^{2} y
$$

where $\hat{f}$ is the Fourier transform of $\zeta_{t}^{0}$. Since $\alpha$ is smooth, $\hat{f}$ decays rapidly. We also have

$$
\left|\chi_{\kappa}(p)-\chi_{\kappa}(q)\right| \leqq 2 \frac{|p-q|}{\kappa^{2}}\left((|p|+\kappa) \chi_{\kappa}(p)+(|q|+\kappa) \chi_{\kappa}(q)\right),
$$

and thus $F_{\kappa, t}$ is $O(1 / \kappa)$.

We are left with (a). First we note that

$$
\left.\operatorname{det}_{3}\left[1+S \zeta_{t}^{0}\right] \exp E(t)\right|_{t=0}=1 .
$$

To prove (a) we will show that

so that

$$
\frac{\partial}{\partial t} \log \left(\operatorname{det}_{3}\left[1+S \zeta_{t}^{0}\right] \exp E(t)\right)=\frac{-t}{2 \pi}\|\partial \alpha\|_{2}^{2}
$$

$$
\left.\operatorname{det}_{3}\left[1+S \zeta_{t}^{0}\right] \exp E(t)\right|_{t=1}=\frac{-1}{4 \pi}\|\partial \alpha\|_{2}^{2}
$$

as needed.

Now

$$
\begin{aligned}
\lim _{\kappa \rightarrow \infty} & \frac{\partial}{\partial t}\left(\operatorname{det}_{3}\left[1+S \zeta_{t}^{0}\right] \exp \operatorname{Tr} \Xi_{\kappa}\left(S \zeta_{t}^{0}-\left(S \zeta_{t}^{0}\right)^{2} / 2\right)\right) \\
= & \lim _{\kappa \rightarrow \infty}\left\{\left(\operatorname{Tr} \frac{1}{1+S \zeta_{t}^{0}}\left(S \zeta_{t}^{0}\right)^{2} S\left(\zeta_{t}^{0}\right)^{\prime}+\operatorname{Tr} S_{\kappa}\left(\zeta_{t}^{0}\right)^{\prime}-\operatorname{Tr} S_{\kappa} \zeta_{t}^{0} S\left(\zeta_{t}^{0}\right)^{\prime}\right)\right. \\
& \left.\cdot \operatorname{det}_{3}\left[1+S \zeta_{t}^{0}\right] \exp E_{\kappa, t}\right\} \\
= & \lim _{\kappa \rightarrow \infty}\left\{\operatorname{Tr}\left(\frac{1}{1+S \zeta_{t}^{0}} S_{\kappa}\left(\zeta_{t}^{0}\right)^{\prime}+S \zeta_{t}^{0} S_{\kappa}\left(\zeta_{t}^{0}\right)^{\prime}-S_{\kappa} \zeta_{t}^{0} S\left(\zeta_{t}^{0}\right)^{\prime}\right)\right.
\end{aligned}
$$




$$
\begin{aligned}
& \left.\cdot \operatorname{det}_{3}\left[1+S \zeta_{t}^{0}\right] \exp E_{\kappa, t}\right\} \\
= & \lim _{\kappa \rightarrow \infty}\left\{\operatorname{Tr}\left(\frac{1}{1+S \zeta_{t}^{0}} S_{\kappa}\left(\zeta_{t}^{0}\right)^{\prime}\right) \operatorname{det}_{3}\left[1+S \zeta_{t}^{0}\right] \exp E_{\kappa, t}\right\}
\end{aligned}
$$

since the difference $\operatorname{Tr}\left[S \zeta_{t}^{0} S_{\kappa}\left(\zeta_{t}^{0}\right)^{\prime}-S_{\kappa} \zeta_{t}^{0} S\left(\zeta_{t}^{0}\right)^{\prime}\right]$ can be estimated in the same fashion as $F_{\kappa, t}$ was in Lemma $2.5(\mathrm{~b})$, and thus vanishes as $\kappa \rightarrow 0$. Explicitly writing out the last line of (2.51) above we see that the derivative (2.51) is equal to

$$
\lim _{\kappa \rightarrow \infty}\left\{\operatorname{det}_{3}\left[1+S \zeta_{t}^{0}\right] \exp E_{\kappa, t} \cdot \operatorname{Tr} U_{t}^{-1} S U_{t} S^{-1} \Xi_{\kappa} S \frac{\partial}{\partial t}\left(U_{t}^{-1} S^{-1} U_{t}\right)\right\} \text {. }
$$

Now

$$
\frac{\partial}{\partial t}\left(U_{t}^{-1} S^{-1} U_{t}\right)=-i \alpha \gamma_{5} U_{t}^{-1} S^{-1} U_{t}+i U_{t}^{-1} S^{-1} U_{t} \alpha \gamma_{5}
$$

so that (using trace cyclicity)

$$
\begin{aligned}
\operatorname{Tr} U_{t}^{-1} S U_{t} S^{-1} \Xi_{\kappa} S \frac{\partial}{\partial t}\left(U_{t}^{-1} S^{-1} U_{t}\right) & =\operatorname{Tr}\left[\Xi_{\kappa}\left(-i \alpha \gamma_{5}\right)+S U_{t} \Xi_{\kappa} U_{t}^{-1} S^{-1}\left(i \alpha \gamma_{5}\right)\right] \\
& =i \operatorname{Tr} S U_{t} \Xi_{\kappa} U_{t}^{-1} S^{-1} \alpha \gamma_{5}
\end{aligned}
$$

since $\gamma_{5}$ is traceless.

Writing

$$
\begin{aligned}
S^{-1} \alpha \gamma_{5} & =\gamma_{5} \gamma_{0}(i \not \partial-1) \alpha \\
& =\gamma_{5}\left(\alpha S^{-1}-2 \gamma_{0} \alpha+i \gamma_{0} \partial \alpha\right)
\end{aligned}
$$

we see that

$$
\operatorname{Tr} S U_{t} \Xi_{\kappa} U_{t}^{-1} S^{-1} \alpha \gamma_{5}=\operatorname{Tr}\left(\Xi_{\kappa} \gamma_{5} \alpha+2 S \gamma_{0} U_{t}^{-1} \Xi_{\kappa} U_{t} \gamma_{5} \alpha-i S \gamma_{0} U_{t}^{-1} \Xi_{\kappa} U_{t} \gamma_{5}(\not \alpha)\right) \text {. }
$$

The first term is clearly zero, and the second term goes to zero as $\kappa \rightarrow \infty$ since it can be bounded, e.g., by $\left\|\mathcal{O}_{\kappa}^{5}\right\|_{3}$. Similar computations with the third term lead to

$$
\frac{\partial}{\partial t}\left(\operatorname{det}_{3}\left[1+S \zeta_{t}^{0}\right] \exp E(t)\right)=\operatorname{det}_{3}\left[1+S \zeta_{t}^{0}\right] \exp E(t) \lim _{\kappa \rightarrow \infty} \operatorname{Tr} S \gamma_{0} \mathcal{O}_{\kappa}^{5}(\partial \alpha)
$$

We can write

$$
\begin{aligned}
\mathcal{O}_{\kappa}^{5}(y, x) & =\frac{i \kappa^{2}}{4 \pi} e^{-\kappa^{2}(x-y)^{2} / 4} \sin t(\alpha(x)-\alpha(y)) \\
& =\frac{-i t \kappa^{2}}{4 \pi} e^{-\kappa^{2}(x-y)^{2} / 4}\left[(y-x) \cdot \partial \alpha+O\left((x-y)^{2}\right)\right],
\end{aligned}
$$

and the singularity of the fermionic covariance is

$$
S \gamma_{0}(x, y) \sim \frac{-i}{2 \pi|x-y|^{2}}\left(x_{\mu}-y_{\mu}\right) \gamma_{\mu}^{E}
$$


so that the trace in (2.57) can be evaluated:

$$
\begin{aligned}
\lim _{\kappa \rightarrow \infty} \operatorname{Tr} S \gamma_{0} \mathcal{O}_{\kappa}^{5}(\partial \alpha)= & \lim _{\kappa \rightarrow \infty} \frac{-t \kappa^{2}}{4 \pi 2} \int d^{2} x d^{2} y e^{-\kappa^{2}(x-y)^{2} / 4} \frac{1}{|x-y|^{2}}[(x-y) \cdot \partial \alpha(x)]^{2} \\
& + \text { higher order terms which vanish } \\
= & \frac{-t}{2 \pi} \int|\partial \alpha|^{2} d^{2} x
\end{aligned}
$$

completing the proof.

Finally, we obtain the following simple estimate on $\operatorname{det}_{3}\left[1+S \gamma_{0}(m(x)-1)\right]$ :

\section{Proposition 2.7.}

$$
\operatorname{det}_{3}\left[1+S \gamma_{0}(m(x)-1)\right] \leqq \exp \left[2\left|\Lambda_{-1}\right| / \pi+O\left(\left|\partial \Lambda_{-1}\right|\right)\right]
$$

Proof. Let $\tilde{K}=S \zeta$. In view of Proposition 2.2 , we need only estimate

$$
\begin{aligned}
\left|\operatorname{det}_{3}(1+\tilde{K})\right| & =\left|\operatorname{det}_{4}(1+\tilde{K}) \exp \left[-\operatorname{Tr} \tilde{K}^{4} / 4\right]\right| \\
& \leqq \exp \text { const }\|\tilde{K}\|_{4}^{4} \\
& \leqq \exp O\left(\left|\partial \Lambda_{-1}\right|\right)
\end{aligned}
$$

by Lemma 5.4 .

\section{The Cluster Expansion}

Let us consider the Fredholm minor

$$
\operatorname{det}_{j k}\left(g_{j},\left[\gamma_{0}(i \not \partial+m(x))\right]^{-1} h_{k}\right)_{L^{2}} \operatorname{det}_{3}\left[1+S \gamma_{0}(m(x)-1)\right]
$$

where $g_{j}, h_{k} \in C_{0}^{\infty}\left(\mathbb{R}^{2}\right)$. The reduced Green's function of Theorem 1 corresponds to the special case where there are only two test functions, denoted $g, h$.

Now the expression (3.1) is clearly equal to

$$
\operatorname{det}_{j k}\left(g_{j}, U^{-1}[1+S \zeta]^{-1} S U h_{k}\right)_{L^{2}} \operatorname{det}_{3}[1+S \zeta] \frac{\operatorname{det}_{3}\left[1+S \gamma_{0}(m(x)-1)\right]}{\operatorname{det}_{3}[1+S \zeta]} .
$$

The ratio of regularized determinants was calculated in Lemma 2.2, and is equal to

$$
\exp \left[2\left|\Lambda_{-1}\right| / \pi+O\left(\left|\partial \Lambda_{-1}\right|\right)\right]
$$

hence we will only need to perform the cluster expansion on the Fredholm minor

$$
\operatorname{det}_{j k}\left(g_{j}, U^{-1}[1+S \zeta]^{-1} S U h_{k}\right)_{L^{2}} \operatorname{det}_{3}[1+S \zeta] .
$$

It will simplify the analysis considerably to work in $\mathscr{H}^{\prime}=L^{2}\left(\mathbb{R}^{2}\right) \oplus L^{2}\left(\mathbb{R}^{2}\right)$ instead of $\mathscr{H}=\mathscr{H}_{-1 / 2} \oplus \mathscr{H}_{-1 / 2}$. This results in replacing the operator $\widetilde{K}=S \zeta$ by $D^{1 / 2} S \zeta D^{-1 / 2}$, where $D=(-\Delta+1)^{1 / 2}$. Thus we have

$$
\begin{aligned}
& \operatorname{det}_{j k}\left(g_{j}, U^{-1}[1+S \zeta]^{-1} S U h_{k}\right)_{L^{2}} \operatorname{det}_{3}[1+S \zeta] \\
& \quad=\operatorname{det}_{j k}\left(\tilde{g}_{j},[1+S \zeta]^{-1} S \tilde{h}_{k}\right)_{L^{2}} \operatorname{det}_{3}[1+S \zeta] \\
& \quad=\operatorname{det}_{j k}\left(D^{-1 / 2} \tilde{g}_{j},\left[1+D^{1 / 2} S \zeta D^{-1 / 2}\right]^{-1} D^{1 / 2} S \tilde{h}_{k}\right)_{L^{2}} \operatorname{det}_{3}\left[1+D^{1 / 2} S \zeta D^{-1 / 2}\right],
\end{aligned}
$$


with

$$
\tilde{g}_{j}=U^{-1} g_{j}, \quad \tilde{h}_{\mu}=U h_{k} .
$$

We wish to express the Fredholm minor (3.4) as a trace of an antisymmetric product of $[1+S \zeta]^{-1}$ multiplied by an operator on $\wedge^{k} \mathscr{H}^{\prime}$. This structure will be maintained throughout, and is necessary in order to obtain the proper estimates on the terms produced by differentiation. We write the expression (3.5) as

$$
\mathscr{J} ! \operatorname{Tr} \operatorname{H\mathscr {H}}^{\prime}\left(\bigwedge_{j=1}^{\mathscr{J}}\left[1+D^{1 / 2} S \zeta D^{-1 / 2}\right]^{-1} P_{j}\right) \operatorname{det}_{3}\left[1+D^{1 / 2} S \zeta D^{-1 / 2}\right],
$$

where $P_{j}$ is the operator on $\mathscr{H}^{\prime}$ given by

$$
P_{j} \equiv\left(D^{-1 / 2} \tilde{g}_{j}, \cdot\right) D^{1 / 2} S \tilde{h}_{j} .
$$

We now introduce decoupling parameters on the bonds of the lattice $l \mathbb{Z}^{2}$; for all $s_{b}=1$ we have the same equation as before, while we have complete decoupling across bonds with $s_{b}=0$. We only decouple bonds that are far away $(O(l)$ - the specific distance will be chosen later) from regions where the mass is changing, i.e. far from the region where $\alpha(x)$ is changing. We denote the subset of bonds on which we decouple by $\mathscr{B}(\alpha)$.

We use the decoupling method of Balaban and Gawędzki [1], with $\Delta$ on the $l$-lattice. Let $\Delta, \Delta^{\prime}$ be $l$-lattice squares, and take $s \in[0,1]^{\mathscr{B}(\alpha)}$. Then define

$$
H\left(s, \Delta, \Delta^{\prime}\right)=\sum_{\text {finite } \gamma \subset \mathscr{B}(\alpha)} \prod_{b \in \gamma} s_{b} \prod_{b \notin \gamma}\left(1-s_{b}\right) \frac{\bar{C}^{\mathscr{B}(\alpha) \backslash \gamma}\left(\Delta, \Delta^{\prime}\right)}{\bar{C}\left(\Delta, \Delta^{\prime}\right)},
$$

where

$$
\bar{C}^{\Gamma}\left(\Delta, \Delta^{\prime}\right)=\int_{\Delta} d x \int_{\Delta^{\prime}} d y C^{\Gamma}(x, y), \quad C^{\Gamma}=\left(-\Delta_{\Gamma}^{D}+m_{c}^{2}\right)^{-1}
$$

$\Delta_{\Gamma}^{D}$ is the Laplacian with Dirichlet boundary conditions on $\Gamma$ and $m_{c}>0$ is a sufficiently small constant to be chosen later.

Definition 3.1. Let $A$ be an operator on $\mathscr{H}^{\prime}$. Then we define

$$
A_{s} \equiv \sum_{\Delta} \chi_{\Delta} A \chi_{\Delta}+\sum_{\Delta \neq \Delta^{\prime}} H\left(s, \Delta, \Delta^{\prime}\right) \chi_{\Delta} A \chi_{\Delta^{\prime}}
$$

The decoupled version of Eq. (3.7) is

where

$$
\mathscr{J} ! \operatorname{Tr}\left(\bigwedge_{j=1}^{\mathscr{J}}[1+K(s)]^{-1} P_{j}\right) \operatorname{det}_{3}[1+K(s)] \equiv Z(s),
$$

and where

$$
K(s) \equiv\left(D^{1 / 2} S D^{1 / 2}\right)_{s}\left(D^{-1 / 2}\right)_{s} \zeta\left(D^{-1 / 2}\right)_{s}
$$

$$
P_{j}(s) \equiv\left(\left(D^{-1 / 2}\right)_{s} \tilde{g}_{j}, \cdot\right)\left(D^{1 / 2} S\right)_{s} \tilde{h}_{j} .
$$

Note that $Z(s)$ factors on connected regions whose boundary consists of bonds with $s_{b}=0$.

The Glimm-Jaffe-Spencer $[5,6]$ cluster expansion is essentially an application of the fundamental theorem of calculus. For a partially decoupled function $F(s)$ 
we have

where

$$
F(1)=\sum_{\Gamma \in \mathscr{B}(\alpha)} \int_{0}^{1} d s_{\Gamma} \partial_{s}^{\Gamma} F(s)
$$

$$
\partial_{s}^{\Gamma}=\prod_{b \in \Gamma} \partial_{s_{b}}, \quad d s_{\Gamma}=\prod_{b \in \Gamma} d s_{b}
$$

and we set $s_{b}=0$ for all $b \notin \Gamma$. We can put this all together with the following:

Proposition 3.2 (Cluster Expansion). The correlation function (3.11) satisfies the following convergent expansion:

$$
Z(\mathbf{1})=\sum_{\Gamma \in \mathscr{B}(\alpha)} \int d s_{\Gamma} \partial_{s}^{\Gamma} Z(s)
$$

and for some constants $c$ and $\delta^{\prime}$ obeys the bound

$$
|Z(\mathbf{1})| \leqq c^{\mathscr{J}} e^{-\delta^{\prime} d\left(\left\{g_{J}\right\},\left\{h_{k}\right\}\right)} e^{O\left(\left|\partial \Lambda_{-1}\right|\right)} .
$$

The remainder of this paper will be dedicated to proving the above proposition; in addition to the factoring across bonds with $s_{b}=0$ the main fact we will need is a bound on cluster activities that is exponential in the size of the cluster.

A typical term in our expansion will have the form

$$
k ! \operatorname{Tr}\left(\wedge^{k}[1+K(s)]^{-1} \cdot G\right) \operatorname{det}_{3}[1+K(s)] \equiv \tau_{k}(G),
$$

where $G$ is an operator on $\wedge^{k} \mathscr{H}^{\prime}$ given by antisymmetric products of operators of the type $A, E$ and $P$ defined below. The result of performing one differentiation on $\tau_{k}(G)$ is then [3]

where

$$
\frac{\partial}{\partial s} \tau_{k}(G)=\tau_{k}\left(\frac{\partial G}{\partial s}\right)+\tau_{k+1}\left(G \wedge A_{s}\right)-\tau_{k}\left(G \cdot d \wedge{ }^{k} E_{s}\right),
$$

$$
A_{s}=K^{2}(s) \frac{\partial K(s)}{\partial s}, \quad E_{s}=(1-K(s)) \frac{\partial K(s)}{\partial s}
$$

and the operator $d \wedge^{k}$ identifies $E_{s}$ with an element of $\wedge^{k} \mathscr{H}^{\prime}$ :

$$
d \wedge{ }^{k} E=k E \wedge \mathbf{I}^{k-1}, \quad \mathbf{I}^{k-1}=1 \wedge \underset{k-1 \text { times }}{\cdots} \wedge 1 .
$$

We can now write down the generic term in the cluster expansion,

$$
\begin{aligned}
\partial_{s}^{\Gamma} Z(s) & =\partial_{s}^{\Gamma} \tau_{\mathscr{J}}\left(\bigwedge_{j=1}^{\mathscr{J}} P_{j}(s)\right) \\
& =\sum_{\pi \in \mathscr{P}(\Gamma)} \sum_{\text {decomp } \pi} \partial^{\pi_{0}} \tau_{r}\left(P\left(\pi_{P}\right) \wedge A\left(\pi_{A}\right) \cdot d \wedge^{r} E\left(\pi_{E}\right)\right) .
\end{aligned}
$$

Here the set of derivatives that have been applied to the Fredholm minor is decomposed according to whether they result in a factor of type $A, E$ or $P$, or produce a higher derivative of such a term, as follows:

$$
\pi=\pi_{0} \cup \pi_{P} \cup \pi_{A} \cup \pi_{E}
$$


and $\mathscr{P}(\Gamma)$ denotes the set of partitions of $\Gamma$. The other terms appearing in (3.22) are:

$$
P\left(\pi_{P}\right)=\left(\prod_{\gamma \in \pi_{P}} \partial_{s}^{\gamma}\right) P_{1}(s) \wedge \cdots \wedge P_{\mathscr{J}}(s)
$$

(where each $\partial_{s}^{\gamma}$ acts on a different $P_{i}(s)$ ),

$$
\begin{gathered}
A\left(\pi_{A}\right)=\wedge_{\gamma \in \pi_{A}} K^{2}(s) \partial_{s}^{\gamma} K(s), \\
d \wedge^{r} E\left(\pi_{E}\right)=\prod_{\gamma \in \pi_{E}} d^{\prime} \wedge^{r}(1-K(s)) \partial_{s}^{\gamma} K(s),
\end{gathered}
$$

where $r=\mathscr{J}+\left|\pi_{A}\right|$ and the $d^{\prime} \wedge$ means that terms where $E$ derivatives precede $A$ derivatives (according to an arbitrary ordering of the bonds) are omitted, and finally

$$
\partial^{\pi_{0}}=\prod_{\gamma \in \pi_{0}} \partial_{s}^{\gamma}
$$

each $\partial_{s}^{\gamma}$ acts on an undifferentiated $K(s)$ in an $A$ or $E$ factor.

We will be estimating (3.22) by fixing all the localization squares in the decoupling (Definition 3.1). To indicate a term so localized we write $A_{l}, E_{l}$, etc. We re-write (3.22) to make this explicit:

Lemma 3.3. The derivative $\partial_{s}^{\Gamma} Z(s)$ may be written as the following sum over localized terms:

$$
\partial_{s}^{\Gamma} Z(s)=\sum_{\pi \in \mathscr{P}(\Gamma)} \sum_{\text {decomp } \pi} \sum_{\substack{\text { localizations } \\\left\{\Delta_{i}\right\}}} \partial^{\pi_{0}} \tau_{r}\left(P_{l}\left(\pi_{P}\right) \wedge A_{l}\left(\pi_{A}\right) \cdot d \wedge^{r} E_{l}\left(\pi_{E}\right)\right) .
$$

\section{Combinatoric Estimates}

We will estimate the sums in Lemma 3.3, primarily using the following lemma:

Lemma 4.1 (Method of Combinatoric Factors). Given two sequences $\left\{a_{n}\right\}$ and $\left\{c_{n}\right\}$, if $\sum_{n}\left|c_{n}\right|^{-1}<1$, then

$$
\sum_{n}\left|a_{n}\right| \leqq \sup \left|c_{n}\right|\left|a_{n}\right|
$$

First we see that

$$
\left|\partial_{s}^{\Gamma} Z(s)\right| \leqq \sum_{\pi} 4^{|\pi|} \sup _{\text {decomp localizations }}\left|\partial^{\pi_{0}} \tau_{r}\left(P_{l} \wedge A_{l} \cdot d^{\prime} \wedge^{r} E_{l}\right)\right| .
$$

We can localize the $A$ factors (this means choosing a particular square from each of the sums over characteristic functions implicit in the definition of the decoupling) with a combinatoric factor

$$
O(1)|\gamma|^{O(1)} \prod_{k} \exp \varepsilon d\left(\Delta_{k}, \gamma\right)
$$

since

$$
\frac{1}{|\gamma|} \sum_{\Delta} \exp [-\varepsilon d(\Delta, \gamma)]<O(1) \text { for } \quad \varepsilon l>1
$$


Similarly we can localize the $P$ factors with combinatoric factors

$$
O(1) \exp \varepsilon\left[d\left(\Delta_{j}, \bar{\Delta}_{j}\right)+d\left(\Delta_{j}^{\prime}, \bar{\Delta}_{j}^{\prime}\right)\right]
$$

where $\bar{\Delta}_{j}$ and $\bar{\Delta}_{j}^{\prime}$ are the supports of the test functions $h_{j}$ and $g_{j}$.

For the $E$ factors, in addition to fixing a localization we wish to fix a choice of terms in the exterior derivative. For the localization we proceed just as we did for the $A$ factors, yielding a combinatoric factor like (4.2).

Combining the above we get the following:

\section{Proposition 4.2.}

$$
\begin{aligned}
& \left|\partial_{s}^{\Gamma} Z(s)\right| \leqq \sum_{\pi} O(1)^{|\Gamma|} \sup _{\begin{array}{c}
\text { decomps, } \\
\text { localizations }
\end{array}}\left|\partial^{\pi_{0}} \tau_{r}\left(P_{l} \wedge A_{l} \cdot d^{\prime} \wedge^{r} E_{l}\right)\right| \\
& \cdot \prod_{\gamma \in \pi_{A} \cup \pi_{E}}\left(O(1)|\gamma|^{O(1)} \prod_{k} \exp \varepsilon d\left(\Delta_{k}, \gamma\right)\right) \\
& \cdot \prod_{j=1}^{\mathscr{f}} O(1) \exp \varepsilon\left[d\left(\Delta_{j}, \bar{\Delta}_{j}\right)+d\left(\Delta_{j}^{\prime}, \bar{\Delta}_{j}^{\prime}\right)\right] .
\end{aligned}
$$

We now want to use additional combinatoric factors to restrict the internal structure of the $A, P$ and $E$ terms. These estimates involve exponential pinning; see for example [1] p. 301. To fix a term in the exterior derivative, let $e_{L}(\Delta)$ be the number of $E$ terms with left-most localization square $\Delta$. Then the number of terms is bounded by

$$
2^{\mathscr{f}+|\Gamma|} \prod_{\Delta} e_{L}(\Delta) ! \leqq 2^{\mathscr{f}} O(1)^{|\Gamma|} \prod_{\gamma \in \pi_{E}} \exp \varepsilon d\left(\gamma, \Delta_{L}\right),
$$

where $\Delta_{L}$ is the left-most localization square corresponding to the $E$ factor being differentiated (by $\gamma \ni b$ ). Now we consider restricting the sum over the $P$ derivatives. There are $\mathscr{J}$ factors and $\left|\pi_{P}\right|$ derivatives, so there are less than $(2 \mathscr{J})^{\left|\pi_{P}\right|}$ possibilities (the 2 comes from the choice of the bra or ket). We have the estimate

$$
(2 \mathscr{J})^{\left|\pi_{P}\right|} \leqq O(1)^{\left|\pi_{P}\right|+\mathscr{g}} \prod_{\gamma \in \pi_{P}} \exp \left[\varepsilon \min \left\{d\left(\gamma, \Delta_{l}\right), d\left(\gamma, \bar{\Delta}_{l}\right)\right\}\right],
$$

where $\Delta_{l}$ is the localization square corresponding to $\gamma$ chosen via (4.4) and the choice of the bra or ket, and $\bar{\Delta}_{l}$ is the corresponding test function square. We also restrict our choice for the distribution of derivatives of $\pi_{0}$. There are less than $\left[O(1)\left(\left|\pi_{A}\right|+\left|\pi_{E}\right|\right)\right]^{\left|\pi_{0}\right|}$ factors, so we get an estimate similar to (4.6),

$$
\left[O(1)\left(\left|\pi_{A}\right|+\left|\pi_{E}\right|\right)\right]^{\left|\pi_{0}\right|} \leqq O(1)^{|\pi|} \prod_{\gamma \in \pi_{0}} \exp \left[\varepsilon \min \left\{d\left(\gamma, \Delta_{l}\right), d\left(\gamma, \Delta_{l}^{\prime}\right)\right\}\right],
$$

where $\Delta_{l}$ and $\Delta_{l}^{\prime}$ are the localization squares chosen via (4.2) or its equivalent that surround the factor being differentiated by $\gamma$.

Finally, because each $K(s)$ has three decouplings each term can actually be differentiated a number of ways, so that the partitions $\pi_{A}, \pi_{E}$ and $\pi_{0}$ must be divided into sub-partitions. However, this simply gives a factor of $O(1)^{|\Gamma|}$, and we will leave our notation unchanged for the time being.

The above discussion can be summarized by the following proposition: 


\section{Proposition 4.3.}

$$
\begin{aligned}
\left|\partial_{s}^{\Gamma} Z(s)\right| \leqq & \sum_{\pi} O(1)^{|\Gamma|+\mathscr{J}} \sup _{\begin{array}{c}
\text { decomps, } \\
\text { localizations }
\end{array}} \sup _{\begin{array}{c}
\pi_{P} \text {-derivatives } \\
\pi_{0} \text {-derivatives } \\
\text { exter. derivs }
\end{array}}\left|\partial^{\pi^{\prime}} \tau_{r}\left(P_{l}^{\prime} \wedge A_{l}^{\prime} \cdot d^{\prime} \wedge{ }^{r} E_{l}^{\prime}\right)\right| \\
& \cdot \prod_{\gamma \in \pi_{A} \cup \pi_{E}}\left(O(1)|\gamma|^{O(1)} \prod_{k} \exp \varepsilon d\left(\Delta_{k}, \gamma\right)\right) \\
& \cdot \prod_{k=1}^{\mathscr{J}} O(1) \exp \varepsilon\left[d\left(\Delta_{k}, \bar{\Delta}_{k}\right)+d\left(\Delta_{k}^{\prime}, \bar{\Delta}_{k}^{\prime}\right)\right] \\
& \cdot \prod_{\gamma \in \pi_{E}} \exp \varepsilon d\left(\gamma, \Delta_{L}\right) \prod_{\gamma \in \pi_{P}} \exp \left[\varepsilon \min \left\{d\left(\gamma, \Delta_{l}\right), d\left(\gamma, \bar{\Delta}_{l}\right)\right\}\right] \\
& \cdot \prod_{\gamma \in \pi_{0}} \exp \left[\varepsilon \min \left\{d\left(\gamma, \Delta_{l}\right), d\left(\gamma, \Delta_{l}^{\prime}\right)\right\}\right],
\end{aligned}
$$

where the primes on $\pi_{0}, P_{l}, A_{l}$ and $d^{\prime} \wedge^{r} E_{l}$ indicate that we take only one term from each summation.

\section{Estimates on Kernels}

In order to prove convergence of the cluster expansion we must now prove analytical estimates on the expressions $\left|\partial^{\pi_{0}^{\prime}} \tau_{r}\left(P_{l}^{\prime} \wedge A_{l}^{\prime} \cdot d^{\prime} \wedge{ }^{r} E_{l}^{\prime}\right)\right|$ appearing in Proposition 4.3 above. Each of these terms is of the form

$$
r ! \operatorname{Tr}\left(\wedge^{r}[1+K(s)]^{-1}, Q_{1}^{\gamma_{1}} \wedge Q_{2}^{\gamma_{2}} \wedge \cdots \wedge Q_{r}^{\gamma_{r}}\right) \operatorname{det}_{3}[1+K(s)]
$$

where each $Q_{i}^{\gamma_{i}}$ takes the form

or

$$
Q_{i}^{\gamma_{1}}=A^{\gamma_{i}^{1}} E^{\gamma_{i}^{2}} E^{\gamma_{1}^{3}} \cdots E^{\gamma_{i}^{k}}
$$

$$
Q_{i}^{\gamma_{1}}=P^{\gamma_{i}^{1}} E^{\gamma_{i}^{2}} E^{\gamma_{i}^{3}} \cdots E^{\gamma_{t}^{k_{2}}}
$$

and each $A, P$ or $E$ is fully localized as indicated above.

Let

$$
G_{1}(\gamma, \delta) \equiv \sum_{\sigma \in S_{|;|}} \exp -\delta\left|l_{\sigma}(\gamma)\right|
$$

where $S_{n}$ denotes the permutation group on $n$ elements, and where the "size" $\left|l_{\sigma}(\gamma)\right|$ of the linear ordering of $\gamma$ determined by the permutation $\sigma \in S_{|\gamma|}$ is defined in [3], page 12 , and let

$$
G_{P}\left(Q_{i}^{\gamma_{1}}\right)= \begin{cases}1, & \text { if } Q_{i}^{\gamma_{t}} \text { is of the form (5.2) } \\ \exp \left(-\delta_{6} \sum_{i} d\left(\Delta_{i j}^{1}, \bar{\Delta}_{i}\right)+d\left(\Delta_{i j}^{2}, \bar{\Delta}_{i}^{\prime}\right)\right), & \text { if } Q_{i}^{\gamma_{\mathrm{t}}} \text { is of the form (5.3) }\end{cases}
$$

where $\Delta_{i j}^{1}, \Delta_{i j}^{2}$ are the localizations associated with the $P$ factors.

The required estimate is as follows:

Proposition 5.1. Choose $m_{c}$, the decoupling mass in (3.10), sufficiently small. Then 
there exist $\delta_{1}, \ldots, \delta_{6}>0$ such that (uniformly in $s$ )

$$
\begin{aligned}
& r !\left|\operatorname{Tr}\left(\wedge^{r}[1+K(s)]^{-1}, Q_{1}^{\gamma_{1}} \wedge Q_{2}^{\gamma_{2}} \wedge \cdots \wedge Q_{r}^{\gamma_{r}}\right) \operatorname{det}_{3}[1+K(s)]\right| \\
& \leqq O(1) \exp \left(O\left(\left|\partial \Lambda_{-1}\right|\right)\right) \prod_{\left\{\gamma_{2}^{j}\right\}}\left(G_{1}\left(\gamma_{i}^{j}, \delta_{1}\right) \exp \left(-\delta_{2} l\left|\gamma_{i}^{j}\right|\right) \exp \left(-\delta_{3} d\left(\gamma_{i}^{j}, \partial \Lambda_{-1}\right)\right) G_{P}\left(Q_{i}^{\gamma_{i}}\right)\right. \\
& \left.\cdot \exp \left(-\delta_{4} \sum_{k} d\left(\Delta_{i j}^{k}, \gamma_{i}^{j}\right)\right) \exp \left(\delta_{5}\left|\gamma_{i}^{j}\right|\right) \exp (O(l))\right)
\end{aligned}
$$

where $\left\{\Delta_{i j}^{k}\right\}$ are the localization squares associated with the differentiation of $\gamma_{i}^{j}$, and $\gamma_{i}=\bigcup_{j} \gamma_{i}^{j}$.

Note that the $\gamma_{i}^{j}$ correspond to the different ways the derivatives in $\gamma_{i}$ can be applied within each factor, as explained preceding Proposition 4.3. Also, since the $P$ factors do not contribute to decay away from $\partial \Lambda_{-1}$, we indicate by a prime on the product that the $\delta_{3}$ term is absent for differentiations arising from $P$ factors.

Proof. We have, using Lemma A.2 of [3],

$$
\begin{aligned}
& r !\left|\operatorname{Tr}\left(\wedge^{r}[1+K(s)]^{-1}, Q_{1}^{\gamma_{1}} \wedge Q_{2}^{\gamma_{2}} \wedge \cdots \wedge Q_{r}^{\gamma_{r}}\right) \operatorname{det}_{3}[1+K(s)]\right| \\
& \quad \leqq\left\|\wedge^{r}[1+K(s)]^{-1} \cdot \operatorname{det}_{3}[1+K(s)]\right\| \prod_{i}\left\|Q_{i}^{\gamma_{i}}\right\|_{1} .
\end{aligned}
$$

We complete the proof with the following two lemmas. The first contains the nonperturbative bound on the determinant; the second is a perturbative bound on the kernels $Q_{i}$.

Lemma 5.2. There exist $c_{1}, c_{2}>0$, independent of $s$, so that

$$
\left\|\wedge^{r}[1+K(s)]^{-1} \cdot \operatorname{det}_{3}[1+K(s)]\right\| \leqq c_{1}^{r} \exp c_{2}\left|\partial \Lambda_{-1}\right| .
$$

Lemma 5.3. For $m_{c}$ sufficiently small the kernels $Q_{i}^{\gamma_{i}}$ satisfy

$$
\begin{aligned}
\left\|Q_{i}^{\gamma_{2}}\right\|_{1} \leqq & \prod_{j} G_{1}\left(\gamma_{i}^{j}, \delta_{1}\right) \exp \left(-\delta_{2} l\left|\gamma_{i}^{j}\right|\right) \exp \left(-\delta_{3} d\left(\gamma_{i}^{j}, \partial \Lambda_{-1}\right)\right) G_{P}\left(Q_{i}^{\gamma_{2}}\right) \\
& \cdot \exp \left(-\delta_{4} \sum_{k} d\left(\Delta_{i j}^{k}, \gamma_{i}^{j}\right)\right) \exp \left(\delta_{5}\left|\gamma_{i}^{j}\right|\right) \exp \left(O\left(\left|\partial \Lambda_{-1}\right|\right)\right) \exp (O(l)),
\end{aligned}
$$

where $\delta_{1} \cdots \delta_{6}>0\left(\delta_{3}=0\right.$ if $\gamma_{i}$ comes from a $P$ factor $)$ are independent of $s$.

The fundamental estimates required to complete the proofs of the above lemmas are the estimates on kernels given in [1]. We quote them here for convenience.

Let

$$
K\left(\Delta, \Delta^{\prime}, \Delta^{\prime \prime}, \Delta^{\prime \prime \prime} ; f\right)=\chi_{\Delta}(-P+1) D^{-1} \chi_{\Delta^{\prime}} D^{-1 / 2} f \chi_{\Delta^{\prime \prime}} D^{-1 / 2} \chi_{\Delta^{\prime \prime \prime}} .
$$

Lemma 5.4 (Proposition A.I.1 of [1]). Let $p>2$. There exists $\varepsilon>0$ such that

$$
\left\|K\left(\Delta, \Delta^{\prime}, \Delta^{\prime \prime}, \Delta^{\prime \prime \prime} ; f\right)\right\|_{p} \leqq\left\|f \chi_{\Delta^{\prime \prime}}\right\|_{L_{\infty}} \exp -\varepsilon\left[d\left(\Delta, \Delta^{\prime}\right)+d\left(\Delta^{\prime}, \Delta^{\prime \prime}\right)+d\left(\Delta^{\prime \prime}, \Delta^{\prime \prime \prime}\right)\right] .
$$

Proof. [1], page 388 . 
Lemma 5.5. Let $H\left(s, \Delta, \Delta^{\prime}\right)$ be given by (3.9). Then

(a) $0 \leqq H\left(s, \Delta, \Delta^{\prime}\right) \leqq 1$,

(b) $\left|\partial_{s}^{\gamma} H\left(s, \Delta, \Delta^{\prime}\right)\right| \leqq \exp (O(l)) \exp \left(2 m_{c} d\left(\Delta, \Delta^{\prime}\right)-\delta m_{c} d\left(\gamma, \Delta, \Delta^{\prime}\right)\right) G_{1}\left(\gamma, \delta_{1}\right) \exp \left(c-\delta_{2} l\right)|\gamma|$.

Proof. [1], page 314.

Proof of Lemma 5.2. By standard determinant estimates, we have

$$
\begin{aligned}
\| & \wedge^{r}[1+K(s)]^{-1} \cdot \operatorname{det}_{3}[1+K(s)] \| \\
& =\left\|\wedge^{r}[1+K(s)]^{-1} \cdot \operatorname{det}_{4}[1+K(s)]\right\| \exp \left[-\operatorname{Tr} K^{4}(s) / 4\right] \\
& \leqq c_{1}^{r} \exp c_{2}\|K(s)\|_{4}^{4} .
\end{aligned}
$$

Now

$$
\begin{aligned}
\|K(s)\|_{4}^{4}= & \operatorname{Tr} K^{*}(s) K(s) K^{*}(s) K(s) \\
= & \sum_{\Delta_{i j} \subset \mathbb{R}^{2}} \operatorname{Tr}\left[K^{*}\left(s ; \Delta_{11}, \Delta_{12}, \Delta_{13}, \Delta_{14} ; \delta\right) K\left(s ; \Delta_{21}, \Delta_{22}, \Delta_{23}, \Delta_{24} ; \delta\right)\right. \\
& \left.\cdot K^{*}\left(s ; \Delta_{31}, \Delta_{32}, \Delta_{33}, \Delta_{34} ; \delta\right) K\left(s ; \Delta_{41}, \Delta_{42}, \Delta_{43}, \Delta_{44} ; \delta\right)\right] \\
& \cdot \prod_{k} H\left(s, \Delta_{k 1}, \Delta_{k 2}\right) H\left(s, \Delta_{k 2}, \Delta_{k 3}\right) H\left(s, \Delta_{k 3}, \Delta_{k 4}\right) \\
\leqq & \sum_{\substack{\Delta_{i j} ;: \Delta_{14}=\Delta_{21}, \Delta_{31}, \Delta_{34}=\Delta_{41}}} \prod_{j}\left\|K\left(s ; \Delta_{j 1}, \Delta_{j 2}, \Delta_{j 3}, \Delta_{j 4} ; \delta\right)\right\|_{4} \\
& \cdot \prod_{k} H\left(s, \Delta_{k 1}, \Delta_{k 2}\right) H\left(s, \Delta_{k 2}, \Delta_{k 3}\right) H\left(s, \Delta_{k 3}, \Delta_{k 4}\right) .
\end{aligned}
$$

Now by Lemma 5.5(a) and Lemma 5.4, this is less than

$$
\begin{aligned}
& \sum_{\substack{\Delta_{i j}: \Delta_{14}=\Delta_{21}, \Delta_{24}=\Delta_{31}, \Delta_{34}=\Delta_{41}}} \prod_{j}\left\|\zeta \chi_{\Delta_{j 2}}\right\|_{L \infty} \exp -\varepsilon\left[d\left(\Delta_{j 1}, \Delta_{j 2}\right)+d\left(\Delta_{j 2}, \Delta_{j 3}\right)+d\left(\Delta_{j 3}, \Delta_{j 4}\right)\right] \\
& \quad \leqq O\left(\left|\partial \Lambda_{-1}\right|\right)
\end{aligned}
$$

since $\operatorname{supp} \zeta \subset \partial \Lambda_{-1}$.

Proof of Lemma 5.3. We estimate

$$
\begin{aligned}
& \left\|A_{l}^{\prime} E_{l}^{\prime} E_{l}^{\prime} \cdots E_{l}^{\prime}\right\|_{1} \leqq\left\|A_{l}^{\prime}\right\|_{1}\left\|E_{l}^{\prime}\right\| \cdots\left\|E_{l}^{\prime}\right\|, \\
& \left\|P_{l}^{\prime} E_{l}^{\prime} E_{l}^{\prime} \cdots E_{l}^{\prime}\right\|_{1} \leqq\left\|P_{l}^{\prime}\right\|_{1}\left\|E_{l}^{\prime}\right\| \cdots\left\|E_{l}^{\prime}\right\| .
\end{aligned}
$$

Recall that

$$
A=\partial^{\gamma_{1}} K \partial^{\gamma_{2}} K \partial^{\gamma_{3}} K,
$$

where $\gamma_{1}, \gamma_{2}$ or both may be empty;

$$
E=(1-K) \partial^{\gamma} K \quad \text { or } \quad E=-\partial^{\gamma_{1}} K \partial^{\gamma_{2}} K,
$$

and $P=P_{j}(s)$ or derivatives thereof. Thus 


$$
\begin{aligned}
\left\|A_{l}^{\prime}\right\|_{1}= & \left\|\sum K\left(s ; \Delta_{1}, \Delta_{2}, \Delta_{3}, \Delta_{4} ; \delta\right) K\left(s ; \Delta_{4}, \Delta_{5}, \Delta_{6}, \Delta_{7} ; \delta\right) K\left(s ; \Delta_{7}, \Delta_{8}, \Delta_{9}, \Delta_{10} ; \delta\right)\right\|_{1} \\
& \cdot \prod_{\substack{\left\{i: \gamma_{1}^{\prime} \cup \gamma^{\prime} \cup \gamma_{3}^{\prime}=\gamma_{1} \\
\gamma_{4}^{\prime} \cup \gamma_{5}^{\prime} \cup \gamma_{6}^{\prime}=\gamma_{2} \\
\gamma_{7}^{\prime} \cup \gamma_{8}^{\prime} \cup \gamma_{9}^{\prime}=\gamma_{3}\right\}}} \partial \gamma^{\gamma_{i}^{\prime}} H\left(s, \Delta_{i}, \Delta_{i+1}\right) \\
\leqq & \sum\left\|K\left(s ; \Delta_{1}, \Delta_{2}, \Delta_{3}, \Delta_{4} ; \delta\right)\right\|_{3}\left\|K\left(s ; \Delta_{4}, \Delta_{5}, \Delta_{6}, \Delta_{7} ; \delta\right)\right\|_{3} \\
& \cdot \prod\left\|K\left(s ; \Delta_{7}, \Delta_{8}, \Delta_{9}, \Delta_{10} ; \delta\right)\right\|_{3}\left|\partial^{\gamma^{\prime}} H\left(s, \Delta_{i}, \Delta_{i+1}\right)\right| .
\end{aligned}
$$

Using Lemma 5.4 and Lemma 5.5(b) this is bounded by

$$
\begin{aligned}
& \exp -\varepsilon\left[d\left(\Delta_{1}, \Delta_{2}\right)+d\left(\Delta_{2}, \Delta_{3}\right)+\cdots+d\left(\Delta_{9}, \Delta_{10}\right)\right] \\
& \cdot \prod \exp \left[2 m_{c} d\left(\Delta_{i}, \Delta_{i+1}\right)-\delta m_{c} d\left(\gamma_{i}^{\prime}, \Delta_{i}, \Delta_{i+1}\right)\right] \\
& \cdot \exp \left(\left(c-\delta_{2} l\right)\left|\gamma_{i}^{\prime}\right|\right) G_{1}\left(\gamma_{i}^{\prime}, \delta_{1}\right) .
\end{aligned}
$$

The $E$ terms are estimated by

$$
\begin{aligned}
\left\|(1-K) \partial^{\gamma} K\right\| & \leqq\left\|\partial^{\gamma} K\right\|+\left\|K \partial^{\gamma} K\right\| \\
& \leqq\left\|\partial^{\gamma} K\right\|_{3}+\left\|K \partial^{\gamma} K\right\|_{3} .
\end{aligned}
$$

Using the same techniques as above, this is bounded by

$$
\begin{aligned}
& \exp -\varepsilon\left[d\left(\Delta_{1}, \Delta_{2}\right)+d\left(\Delta_{2}, \Delta_{3}\right)+\cdots+d\left(\Delta_{7}, \Delta_{8}\right)\right] \\
& \quad \cdot \prod_{\left\{i: \gamma_{1}^{\prime} \cup \gamma_{2}^{\prime} \cup \gamma_{3}^{\prime}=\gamma\right\}} \exp \left[2 m_{c} d\left(\Delta_{i}, \Delta_{i+1}\right)-\delta m_{c} d\left(\gamma_{i}^{\prime}, \Delta_{i}, \Delta_{i+1}\right)\right] \\
& \cdot \exp \left(\left(c-\delta_{2} l\right)\left|\gamma_{i}^{\prime}\right|\right) G_{1}\left(\gamma_{i}^{\prime}, \delta_{1}\right) .
\end{aligned}
$$

Finally, to estimate $\partial^{\gamma} P_{l}^{\prime}$ we note that (recall that the $\tilde{h}$ 's and $\tilde{g}$ 's are localized in blocks $\bar{\Delta}^{\prime}$, and $\gamma=\gamma_{1} \cup \gamma_{2}$ )

$$
\begin{aligned}
\partial^{\gamma} P_{l}^{\prime}(\cdot) & =\left(\chi_{\Delta_{2}} D^{-1 / 2} \tilde{g}_{j}, \cdot\right) \chi_{\Delta_{1}} D^{1 / 2} S \tilde{h}_{j} \partial^{\gamma_{1}} H\left(s, \Delta_{1}, \bar{\Delta}\right) \partial^{\gamma_{2}} H\left(s, \Delta_{2}, \bar{\Delta}^{\prime}\right) \\
& =\left(\widetilde{G}_{j}, \cdot\right) \tilde{H}_{j} \partial^{\gamma_{1}} H\left(s, \Delta_{1}, \bar{\Delta}\right) \partial^{\gamma_{2}} H\left(s, \Delta_{2}, \bar{\Delta}^{\prime}\right),
\end{aligned}
$$

where

$$
\begin{gathered}
\tilde{H}_{j} \equiv \chi_{\Delta_{1}} D^{1 / 2} S \tilde{h}_{j}, \\
\tilde{G}_{j} \equiv \chi_{\Delta_{2}} D^{-1 / 2} \tilde{g}_{j} .
\end{gathered}
$$

Now by straightforward arguments (e.g. Theorem 2.2 of [13])

$$
\begin{aligned}
&\left\|\tilde{H}_{j}\right\|_{\mathscr{H}^{\prime}} \leqq \exp -\varepsilon d\left(\Delta_{1}, \bar{\Delta}\right)\left\|\tilde{h}_{j}\right\|_{\mathscr{H}} \\
&\left\|\tilde{G}_{j}\right\|_{\mathscr{H}^{\prime}} \leqq \exp -\varepsilon d\left(\Delta_{2}, \bar{\Delta}^{\prime}\right)\left\|\tilde{g}_{j}\right\|_{\mathscr{H}}
\end{aligned}
$$

while

$$
\left\|\partial^{\gamma} P\right\|_{1} \leqq\left\|\tilde{H}_{j}\right\|_{\mathscr{H}^{\prime}}\left\|\tilde{G}_{j}\right\|_{\mathscr{H}^{\prime}}\left|\partial^{\gamma_{1}} H\left(s, \Delta_{1}, \bar{\Delta}\right)\right|\left|\partial^{\gamma_{2}} H\left(s, \Delta_{2}, \bar{\Delta}^{\prime}\right)\right| .
$$


Combining (5.22)-(5.24), we get by Lemma 5.5

$$
\begin{aligned}
\left\|\partial^{\gamma} P\right\|_{1} \leqq & \exp \left(2 m_{c}-\varepsilon\right)\left[d\left(\Delta_{1}, \bar{\Delta}\right)+d\left(\Delta_{2}, \bar{\Delta}^{\prime}\right)\right] \\
& \cdot \exp \left[-\delta m_{c}\left(d\left(\gamma_{i}, \Delta_{1}, \bar{\Delta}\right)+d\left(\gamma_{i}, \Delta_{2}, \bar{\Delta}^{\prime}\right)\right)\right] \\
& \cdot\left\|h_{j}\right\|_{\mathscr{H}}\left\|g_{j}\right\|_{\mathscr{H}} \prod_{i=1,2} \exp \left(\left(c-\delta_{2} l\right)\left|\gamma_{i}\right|\right) G_{1}\left(\gamma_{i}, \delta_{1}\right)
\end{aligned}
$$

as needed.

\section{Convergence of the Expansion}

We now want to complete our proof of the convergence of the cluster expansion. First we take $m_{c}$ small so that, for example, $\left(2 m_{c}-\varepsilon\right)<0$ in (5.16), (5.25). Then we take $l$ sufficiently large so that $\left(c-\delta_{2} l\right)<0$ in (5.16) and (5.25) with enough left over to beat the $O(1)^{|\Gamma|}$ in Proposition 4.3. Then we can combine Proposition 4.3 and 5.1 to give

$$
\begin{aligned}
\left|\partial_{s}^{\Gamma} Z(s)\right| \leqq & \exp \left(\left(-\delta_{2}^{\prime} d\left(\Delta_{g}, \Delta_{h}\right)\right)\right) \exp \left(O\left(\left|\partial \Lambda_{-1}\right|\right)-\delta_{2}^{\prime}|\Gamma|\right) \\
& \cdot \sum_{\pi} \sup \prod_{\left\{\gamma_{i}^{j}\right\}}^{\prime}\left[G_{1}\left(\gamma_{i}^{j}, \delta_{1}\right) \exp \left(-\delta_{3} d\left(\gamma_{i}^{j}, \partial \Lambda_{-1}\right)\right) \exp (O(l))\right] .
\end{aligned}
$$

Here we consider for simplicity the case of the reduced Green's function, as in Theorem 1, with only two test functions $g, h$ supported in $\Delta_{g}, \Delta_{h}$. The decay between $\Delta_{g}, \Delta_{h}$ can be extracted from the factors in Proposition 5.1, assuming we allow for an increase in $O\left(\left|\partial \Lambda_{-1}\right|\right)$ to compensate for a possible lack of connections going all the way from $\Delta_{g}$ to $\Delta_{h}$.

Let us insert this bound in the starting expansion (3.16), so that we may complete the proof of Proposition 3.2. We obtain

$$
\begin{aligned}
|Z(\mathbf{1})| \leqq & \exp \left(-\delta^{\prime} d\left(\Delta_{g}, \Delta_{h}\right)\right) \exp \left(O\left(\left|\partial \Lambda_{-1}\right|\right)\right) \sum_{\Gamma} \exp \left(-\delta^{\prime}|\Gamma|\right) \sum_{\pi} \sup \prod_{\left\{\gamma_{i}^{j}\right\}}^{\prime} \\
& \cdot\left[\sum_{\sigma} \exp \left(-\delta^{\prime}\left|l_{\sigma}\left(\gamma_{i}^{j}\right)\right|\right) \exp \left(-\delta^{\prime} d\left(\gamma_{i}^{j}, \partial \Lambda_{-1}\right)\right) \exp (O(l))\right],
\end{aligned}
$$

where we have written $G_{1}$ out using (5.4). Now rather than sum over $\pi$ we can just as well sum over all partitions of $\Gamma$ into $\left\{\gamma_{i}^{j}\right\}$ - the original partition $\pi$ can be reconstructed from the structure of differentiations in $\left\{\gamma_{i}^{j}\right\}$. Furthermore, we sum freely over the $\gamma_{i}^{j}$, s, reconstructing $\Gamma$ as their union. To sum over a single $\gamma_{i}^{j}$, we use the estimate

$$
\sum_{\gamma_{i}^{j}} \sum_{\sigma} \exp \left(-\delta^{\prime}\left|l_{\sigma}\left(\gamma_{i}^{j}\right)\right|\right) \exp \left(-\delta^{\prime} d\left(\gamma_{i}^{j}, \partial \Lambda_{-1}\right)\right) \exp (O(l)) \leqq O\left(\left|\partial \Lambda_{-1}\right|\right) .
$$

The first bond yields the factor $O\left(\left|\partial \Lambda_{-1}\right|\right)$, since it is localized to $\partial \Lambda_{-1}$ only. Subsequent bonds are localized by $\left|l_{\sigma}\left(\gamma_{i}^{j}\right)\right|$. The factor $\exp (O(l))$ is beaten by the decay in $d\left(\gamma_{i}^{j}, \partial \Lambda_{-1}\right)$ - for this we choose $\mathscr{B}(\alpha)$ so that $d\left(\mathscr{B}(\alpha), \partial \Lambda_{-1}\right)>O(l)$. This may not be possible at $\Delta_{g}, \Delta_{h}$, however, so there will be two $\exp (O(l))$ factors left over. 
Now in summing freely over $N \gamma_{i}^{j}$, s, we count each partition of $\Gamma N$ ! times. Hence we have an estimate

$$
|Z(\mathbf{1})| \leqq \exp (O(l)) \exp \left(-\delta^{\prime} d\left(\Delta_{g}, \Delta_{h}\right)\right) \exp \left(O\left(\left|\partial \Lambda_{-1}\right|\right)\right) \sum_{N} \frac{1}{N !} O\left(\left|\partial \Lambda_{-1}\right|\right)^{N} .
$$

This completes the proof of Proposition 3.2. It is worth noting that all differentiations were ultimately linked to $\partial \Lambda_{-1}$, there being no interaction away from $\partial \Lambda_{-1}$ after the chiral transformation. Hence it was not necessary to exploit factorization of $Z(s)$ or to compute a ratio of expansions with and without observables.

To complete a proof of Theorem 1, we have only to multiply back the ratio of regularized determinants that we split off in (3.2). This yields the additional factor $\exp \left(2\left|\Lambda_{-1}\right| / \pi\right)$.

\section{Appendix: General Masses}

When $|m(x)|$ takes on values other than 1 , we can prove a result analogous to Theorem 1. We suppose $m(x)$ takes on finitely many values; for definiteness suppose $m(x)$ is real and constant on cubes of some size. The chiral case $m(x)=\mu(x) \exp \left(\mathrm{i} \gamma_{5} \theta(x)\right)$ can be treated similarly. By rescaling we can assume the largest $|m(x)|$ is 1 . Corresponding to each value of $m(x)$ there is a region in $\mathbb{R}^{2}$, where $m(x)$ takes that value. Each of these regions now has a different vacuum energy (but explicitly calculable, as in [7], p. 333), and the bound in Theorem 1 has to be adjusted by the appropriate factors exponential in the volumes. For simplicity we assume $m(x)=1$ at infinity, but the more general case can be handled as well by taking a limit on volumes, normalizing so as to cancel the vacuum energy of the phase at infinity. Constants and decay rates in Theorem 1 will of course not be uniform as the minimum $|m(x)|$ tends towards zero.

The chiral transformation can be performed as in the constant $|m(x)|$ case. We obtain a Fredholm minor as in (3.4), only now the function $\zeta$, as defined by (2.9), no longer vanishes away from suppt $\partial \alpha$. To remedy this, we perform a Neumann series to bring the mass into the propagator $S$. Putting $\delta(x)=\gamma_{0}(1-|m(x)|)$, we can define

$$
S^{\prime}=\left(S^{-1}-\delta\right)^{-1}=S+S \delta S+S \delta S \delta S+\cdots .
$$

This is convergent since $\|S\| \leqq 1$ and $|1-| m(x)|| \leqq \delta_{\max }<1$. Then with $\zeta^{\prime}=\zeta+\delta$ we put $A=S^{\prime} \zeta^{\prime}, B=-S \delta, C=A+B+A B=S \zeta$ and by Lemma 2.3,

$$
\operatorname{det}_{3}[1+S \zeta]=\operatorname{det}_{3}\left[1+S^{\prime} \zeta^{\prime}\right] \operatorname{det}_{3}[1-S \delta] \exp (\operatorname{Tr} T)
$$

where

$$
T=(A B)^{2} / 2+A B^{2}+B A^{2} .
$$

As in [7], Sect. 5.5, we can analyze the series for $\log \operatorname{det}_{3}[1-S \delta]+\operatorname{Tr} T$ and break it into a sum over the values of $m(x)$ of vacuum energies $\times$ volumes, with corrections associated with the length of boundary.

It remains to analyze $\operatorname{det}_{3}\left[1+S^{\prime} \zeta^{\prime}\right]$ and the corresponding minors with a cluster expansion. The new perturbation $\zeta^{\prime}$ vanishes except near boundary regions. The 
only essential new feature is the interpolation of $S^{\prime}$. By analogy with (3.12) we put

$$
K(s)=\left(D^{1 / 2} S D^{1 / 2}\right)_{s}\left(D^{-1 / 2}\right)_{s}\left(\sum_{n=0}^{\infty}(\delta S)^{n}\right)_{s} \zeta^{\prime}\left(D^{-1 / 2}\right)_{s^{*}}
$$

Note that in contrast with [7], we decouple after the mass shift. While this is inconvenient for some purposes it does enable us to get the necessary propagator estimates. Specifically, the new estimates required are on $\left\|\chi_{\Delta}(\delta S)^{n} \chi_{\Delta^{\prime}}\right\|$. Using the Combes-Thomas method [2], we conjugate $\delta S$ by $\exp (\eta x)\left(x=x_{0}\right.$ or $\left.x_{1}\right)$. For small enough $\eta$ the resulting operator still has norm less than 1. (Near the diagonal the conjugation is multiplication by $1+O(\eta)$, while far from the diagonal the decay of $S$ takes over.) Hence the series converges and a decay $\exp \left(-O(\eta) d\left(\Delta, \Delta^{\prime}\right)\right)$ can be extracted.

\section{References}

1. Balaban, T., Gawedzki, K.: A low temperature expansion for the pseudoscalar Yukawa theory of quantum fields in two spacetime dimensions. Ann Inst. H. Poincaré, Section A: 36, 271 (1982)

2. Combes, J., Thomas, L.: Asymptotic behavior of eigenfunctions for multiparticle Schröedinger operators. Commun. Math. Phys. 34, 251-270 (1973)

3. Cooper, A., Rosen, L.: The weakly coupled Yukawa ${ }_{2}$ field theory: Cluster expansion and Wightman axioms. Trans. Am. Math. Soc. 234, 1 (1977)

4. Glimm, J., Jaffe, A.: Quantum physics, Berlin, Heidelberg, New York: Springer, 1987

5. Glimm, J., Jaffe, A., Spencer, T.: The particle structure of the weakly coupled $P(\phi)_{2}$ model and other applications of high temperature expansions. Part I: Physics of Quantum Field Models, Part II: The cluster expansion. In: Constructive Quantum Field Theory (Erice 1973). Velo, G., Wightman, A. S. (eds.) Berlin, Heidelberg, New York: Springer 1973

6. Math. 100, 585 (1974)

7. Imbrie, J. Z.: Phase diagrams and cluster expansions for low temperature $\mathscr{P}(\phi)_{2}$ models. Commun. Math. Phys. 82, 261-343 (1981).

8. Janowsky, S., Weitsman, J.: The phase structure of the two-dimensional $N=2$ Wess-Zumino model. Harvard University preprint HUTMP 90/B73; Janowsky, S.: Harvard University Thesis (1990)

9. Jaffe, A., Lesniewski, A.: A Priori Estimates for $N=2$ Wess-Zumino Models on a cylinder. Commun. Math. Phys. 114, 553 (1988)

10. Jaffe, A., Lesniewski, A., Weitsman, J.: Index of a family of Dirac operators on loop space. Commun. Math. Phys. 112, 75 (1987)

11. - : The two-dimensional, $N=2$ Wess-Zumino models on a cylinder. Commun. Math. Phys. 114, 147 (1988)

12. —— The loop space $S^{1} \rightarrow \mathbb{R}$ and supersymmetric quantum fields. Ann. Phys. 183, 337 (1988)

13. Seiler, E., Simon, B.: Bounds on the Yukawa ${ }_{2}$ quantum field theory: upper bound on the pressure, Hamiltonian bound and linear lower bound. Commun. Math. Phys. 45, 99 (1975)

14. Simon, B.: Trace ideals and their applications, (London Mathematical Society Lecture Note Series 35) Cambridge: Cambridge University Press 1979

15. Weitsman, J.: A supersymmetric field theory in infinite volume. Harvard University thesis (1988) 
